\title{
Fault Tolerant Control Design Based on Takagi-Sugeno Fuzzy Logic: Application to a Three-Tank System
}

\author{
Himanshukumar R. Patel ${ }^{a}$ and Vipul A. Shah ${ }^{b}$ \\ ${ }^{a}$ Instrumentation \& Control Department, Faculty of Technology, Dharmsinh Desai University, Nadiad, Gujarat,India, \\ e-mail: himanshupatel.ic@ddu.ac.in \\ ${ }^{b}$ Instrumentation \& Control Department, Faculty of Technology, Dharmsinh Desai University, Nadiad, Gujarat,India, \\ e-mail: vashah.ic@ddu.ac.in
}

\begin{abstract}
This paper deals with a novel Fault-Tolerant Control (FTC) strategy for non-linear systems without hardware redundancy in case of actuator and system component failures. These failures correspond to blocking or loss of effectiveness of an actuator, leakage of tank bottom and pipes which could lead to a large degradation in performances. The abrupt and increasing nature faults are introduced into the system, followed by the simulation results. The proposed method is based on a conventional PID controller and T-S Fuzzy controller in order to maintain the acceptable performance subject to faults. The effectiveness of this approach is evaluated through the application to a non-linear pilot plant, a three-tank system. A simulation result is given with IAE and ISE error to illustrate the effectiveness of the proposed approach.
\end{abstract}

Keywords: Abrupt fault, Actuator fault, Fault-tolerant control, Increasing fault, Nonlinear system, System fault, T-S fuzzy control.

\section{Introduction}

At the event of faults in actuator, system components or sensor faults, in conventional feedback control system may result in unacceptable performances. To overcome the limitations of conventional feedback control system, new strategies have been developed since last thirty years, which are proficient of tolerating various faults, during the time still maintaining some required properties of performances [8],[9],[12] and [36]. Research in Fault-Tolerant Control (FTC) seems have a large application domain but it still remains too largely motivated by the controlling of nonlinear system [14],[28],[29],[30]. This fault tolerant op- eration can be realized either passively through the use of robust control law insensitive to faults and actively through a fault detection and diagnosis (FDD) algorithm and the results from FDD controller parameters will reconfigure [23],[25]. The strategy to implement and the level of achieved performance in the event of failures will differ according to the type of process, the allocated degree of freedom and the severity of faults. The FTC methods demonstrates their abilities and effectiveness subject to various faults which involve abrupt and discontinuous variations in the system dynamics. The prime motive of FTC strategy is minimize the loss of productivity (to produce with an acceptable quality) or/and to operate safe operation without risky situation to human operators or to equipments [13],[21].

The paper deals with two serious fault scenarios, one is that loss of effectiveness or partial blocking of actuator. Second fault scenarios is one of the system component (leak $\mathrm{n}$ bottom of the tank) faults occurring in the system. It is difficult to deal with stuck or loss of effectiveness actuator, system component faults because the remaining actuators must compensate for the effects of the failed actuators in the overall system. The main contributions of this paper are as following:

- Proposed unique linearized mathematical model of the three-tank level control process is derived using Takagi Sugeno fuzzy model approach.

- The three-tank level process is converted in piecewise linearized using three different premise variable $z_{1}, z_{2}$, and $z_{3}$, and the membership functions for the premise variable having only two linguistic variable and hence it will not adding additional computational burden for controller also the membership function are not optimized using Genetic Algorithm (GA) which are presented in brief $[5][16]$.

- For designing the proposed Passive FTC controller conventional PID controller and Type-1 
FLC are used, Type-1 FLC structure is easy to implement as compared to Type-2 FLC, but at the same time Type-1 FLC will not incorporate the model uncertainty and noise [15][26].

- The proposed controller structure is simple and effective to control nonlinear process even though the fault occurs, the author of [6] proposed Type-2 fuzzy control system for controlling the longitude of the aircraft.

- The main advantage of the Type-1 FLC is, very less computational burden and no need to apply additional algorithm to convert Type-1 fuzzy sets from Type-2 fuzzy sets [2].

In recent years, there has been an ever increasing push to optimize the controllers to help fulfill the ever increasing demands for high quality products[2]. Overviews on the development of FTCS have been provided in survey articles by [32] and [38], as well as books by [11], [20], [19] and [10]. Most chemical plants are multivariable processes and have to be controlled in order to perform well.The work in [35] suggests a control reconfiguration method based on system responses to accommodate stuck actuators. In recent FTC has design for non-linear system subject to different faults, in [1] and [14].The paper contributed by [17] and [33] has proposed FTC scheme using adaptive fuzzy logic for non-linear system subject to sensor and actuator faults.In [7] author has project Active FTC strategy for three-tank system using analytical redundancy. Passive FTC strategy using artificial intelligence techniques has investigating for interacting and non-interacting level process (single and multiple tank level system) subject to system component (leak) and actuator faults in [21],[22],[24],[25],[28],[29],[30],[31], and excellent results reported in simulation as well as for real-time system.In [18] adaptive fuzzy logic control structure is used to design fault-tolerant control of MIMO nonlinear uncertain systems. The authors of [27],[15] and [5] design Type-2 FLC for the nonlinear level process (MIMO level) process, In [5] and [15] has done comparative results using Type-1 and Type-2 FLC, while in [27] author design interval Type2 TSK FLC for three interconnected conical tank level process, and proved the superiority of the same strategy, however the complexity and computational time is constrain for the controller.

The paper is organized as follows.In section II a general formulation of the problem with mathematical model is given. The proposed FTC approach in this paper is based on the T-S Fuzzy logic control with conventional PID controller described in section III. The simulation results presented in section IV.Finally, a concluding remarks given in section $\mathrm{V}$.

\section{Process Description and Modeling}

The plant dynamic considered in this paper is described by the following m-inputs, n-outputs nonlinear system $\sum$ :

$$
\left(\sum\right):\left\{\begin{array}{c}
\dot{X}=f(X(t))+\sum_{i=1}^{m} g_{i}(X(t)) u_{i}(t) \\
y_{1}(t)=h_{1}(X(t)) \\
\cdots \\
y_{n}(t)=h_{n}(X(t))
\end{array}\right.
$$

Where, $X \in R^{n}$ is the state vector, $U=$ $\left[\begin{array}{lll}u_{1} & \cdots & u_{m}\end{array}\right]^{T} \in R^{m}$ s the control vector, and $Y=\left[\begin{array}{lll}y_{1} & \cdots & y_{n}\end{array}\right]^{T} \in R^{n}$ is the output vector to be controlled. $f(X), g_{i}(X), i=1, \cdot, m$ are smooth vector fields, and $h_{i}(X), i=1, \cdot, m$ are smooth functions, defined on an open set of $R^{n}$.

The physical constraints of the system (1) are defined by:

$$
(\Psi)\left\{\begin{aligned}
X_{\min } & \leq X(t) \leq X_{\max } \\
Y_{\min } & \leq X(t) \leq Y_{\max } \\
U_{\min } \leq X(t) & \leq U_{\max }
\end{aligned}\right.
$$

\subsection{Modeling of Three-tank benchmark system}

In this paper three-tank benchmark system is considered to be controlled. The three-tank system laboratory model can be viewed as a prototype of many industrial applications in process industry, such as chemical and petrochemical plants, oil and gas systems. The typical control issue involved in the system is how to keep the desired liquid level in tank. The principle scheme of the model is shown in Figure 1.The basic apparatus consists of three plexiglass tanks numbered from left to right as 1,2 and 3, with cross sectional area of A. All three tank is identical in size. The process under consideration is a three-tank benchmark system shown in figure 1 .

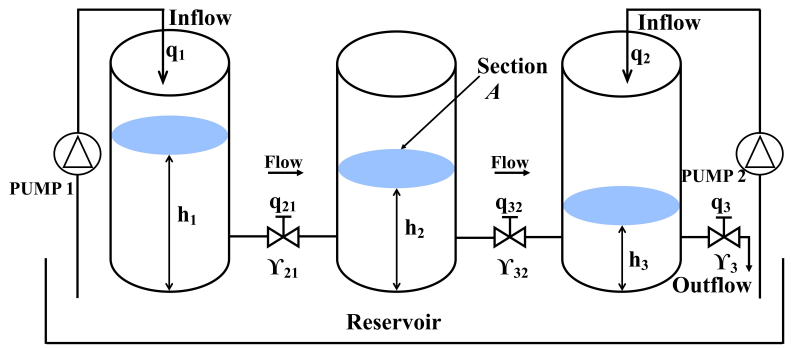

Figure 1: Synoptic of the Three-tank system.

It is composed of three cylindrical tanks with identical cross section A. The tanks are coupled by two con- 
necting cylindrical pipes with a cross section A, and an outflow coefficient $\gamma_{21}=\gamma_{32}$. The nominal outflow is located at the tank 2 , it also has a circular cross section A, and an outflow coefficient $\gamma_{3}$. Two pumps driven by DC motors supply the tanks 1 and 2. Flow meters measure the flows $q_{1}$ and $q_{2}$ provided by each pump. All three tanks are equipped with differential pressure type transducers for measuring the liquid level $\left(h_{1}, h_{2}, h_{3}\right)$. Using the Torricelli rule and the mass balance equations, the system can be represented conveniently by:

$$
\left\{\begin{array}{c}
A * \frac{d h_{1}(t)}{d t}=q_{1}(t)-q_{21}(t) \\
A * \frac{d h_{2}(t)}{d t}=q_{21}(t)-q_{32}(t) \\
A * \frac{d h_{3}(t)}{d t}=q_{2}(t)+q_{32}(t)-q_{3}(t)
\end{array}\right.
$$

where $q_{i j}$ represents the water flow rate from tank tank $j$ to $\left.i\left(i, j=1,2,3 \forall_{i} \neq j\right)\right)$, with

$q_{i j}(t)=\gamma_{i j} A_{n} \operatorname{sign}\left(h_{j}(t)-h_{i}(t)\right) \sqrt{2 * g *\left|h_{j}(t)-h_{i}(t)\right|}$

And $q_{3}$ represents the outflow rate with

$$
q_{3}=\gamma_{3} A_{n} \sqrt{2 g h_{3}(t)}
$$

The system can be written under the non-linear from

$$
\dot{X}(t)=\left[\begin{array}{c}
-\frac{1}{A} * q_{21}(X(t)) \\
-\frac{1}{A}\left\{q_{21}(X(t))-q_{32}(X(t))\right\} \\
-\frac{1}{A}\left\{q_{32}(X(t))-q_{3}(X(t))\right\}
\end{array}\right]+\left[\begin{array}{cc}
\frac{1}{A} & 0 \\
0 & 0 \\
0 & \frac{1}{A}
\end{array}\right] U(t)
$$

With $X(t)=\left[\begin{array}{lll}h_{1}(t) & h_{2}(t) & h_{3}(t)\end{array}\right]^{T}, U(t)=\left[q_{1}(t)\right]^{T}$, and $Y(t)=\left[h_{3}(t)\right]^{T}$. The liquid level is given in meter and the flows in $\mathrm{m}^{3} / \mathrm{s}$. The set $(\Psi)$ is defined by:

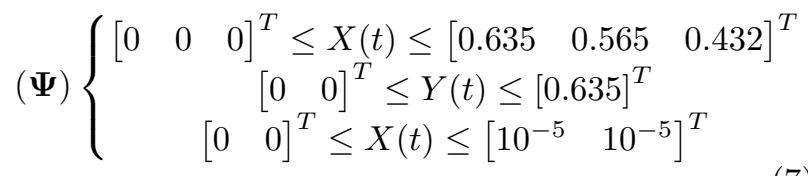

The main objective of the three-tank system is to maintain tank 3 height $h_{3}$ which is controlled variable with manipulated variable inflow $q_{1}$ and $q_{2}$. The standard operating parameters of the three-tank benchmark system presented in table 1 .

\begin{tabular}{|l|l|l|l|}
\hline $\begin{array}{l}\text { Tank area } \\
\left(\mathrm{m}^{2}\right)\end{array}$ & $\begin{array}{l}\gamma_{21} \\
\left(\mathrm{~m}^{3} / \mathrm{sec}\right)\end{array}$ & $\begin{array}{l}\gamma_{32} \\
\left(\mathrm{~m}^{3} / \mathrm{sec}\right)\end{array}$ & $\begin{array}{l}\gamma_{3} \\
\left(\mathrm{~m}^{3} / \mathrm{sec}\right)\end{array}$ \\
\hline 0.615 & 0.9 & 0.8 & 0.3 \\
\hline
\end{tabular}

Table 1: Parameters of the Three-tank process.

\section{$3 \quad$ FTC Strategy using Takagi-Sugeno Fuzzy Logic System}

In order to minimizing the consequence of the actuator and system component faults on proposed threetank benchmark system, we adopt Passive FTC strategy. The proposed controller design in this paper, uses Takagi-Sugeno like fuzzy model. Figure 2 illustrates the overview of proposed robust controller design. The controller consist of conventional PID controller and T-S Fuzzy logic based controller.

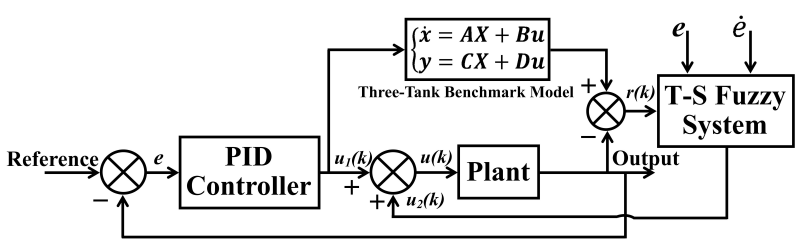

Figure 2: Proposed Passive FTC strategy for the Three-tank system.

\subsection{Takagi-Sugeno fuzzy modeling}

Consider the nonlinear system given in Eq. 3, The goal is to derive a T-S fuzzy model from the nonlinear system given in Eq. 3 by the sector nonlinearity approach as if the response of the T-S fuzzy model in the specified domain exactly match with the response of the original system with the same input $u$. Here $h_{1}, h_{2}$, and $h_{3}$ are nonlinear terms in the Eq. 3 so we make them as our fuzzy variables. Generally they are denoted as $z_{1}, z_{2}, z_{3}$ and are known as premise variables that may be functions of state variables, input variables, external disturbances and/or time. Therefore $z_{1}=x_{1}, z_{2}=x_{2}$ and $z_{3}=x_{3}$.

The first step for any kind of fuzzy modeling is to determine the fuzzy variables and fuzzy sets or so-called membership functions [37].It is assumed in this article that the premise variables are just functions of the state variables for the sake of simplicity. This assumption is needed to avoid a complicated defuzzification process of the fuzzy controllers [2][9].

To acquire membership functions, we calculate the minimum and maximum values of $z_{1}(t), z_{2}(t)$ and $z_{3}(t)$ which under $x_{1} \in[0.235,0.635], x_{2} \in[0.217,0.565]$ and $x_{3} \in[0.147,0.432]$, they are obviously obtained as follows: 


$$
\begin{array}{ll}
\max z_{1}(t)=0.635 & \min z_{1}(t)=0.236 \\
\max z_{2}(t)=0.565 & \min z_{2}(t)=0.217 \\
\max z_{3}(t)=0.432 & \min z_{3}(t)=0.147
\end{array}
$$

Now, $x_{1}, x_{2}$ and $x_{2}$ can be represented by for membership functions $P_{1}, P_{2}, M_{1}, M_{2}, N_{1}$ and $N_{2}$ as follows. as follows:

$$
\begin{gathered}
z_{1}(t)=x_{1}(t)=P_{1}\left(z_{1}(t)\right) \cdot 0.635+P_{2}\left(z_{1}(t)\right) \cdot 0.236, \\
z_{2}(t)=x_{2}(t)=M_{1}\left(z_{2}(t)\right) \cdot 0.565+M_{2}\left(z_{2}(t)\right) \cdot 0.217, \\
z_{3}(t)=x_{3}(t)=N_{1}\left(z_{3}(t)\right) \cdot 0.432+M_{3}\left(z_{3}(t)\right) \cdot 0.147
\end{gathered}
$$

and because $P_{1}, P_{2}, M_{1}, M_{2}, N_{1}$ and $N_{2}$ are actually fuzzy sets according to fuzzy mathematics

$$
\begin{gathered}
P_{1}\left(z_{1}(t)\right)+P_{2}\left(z_{1}(t)\right)=1, \\
M_{1}\left(z_{1}(t)\right)+M_{2}\left(z_{1}(t)\right)=1, \\
N_{1}\left(z_{1}(t)\right)+N_{2}\left(z_{1}(t)\right)=1 .
\end{gathered}
$$

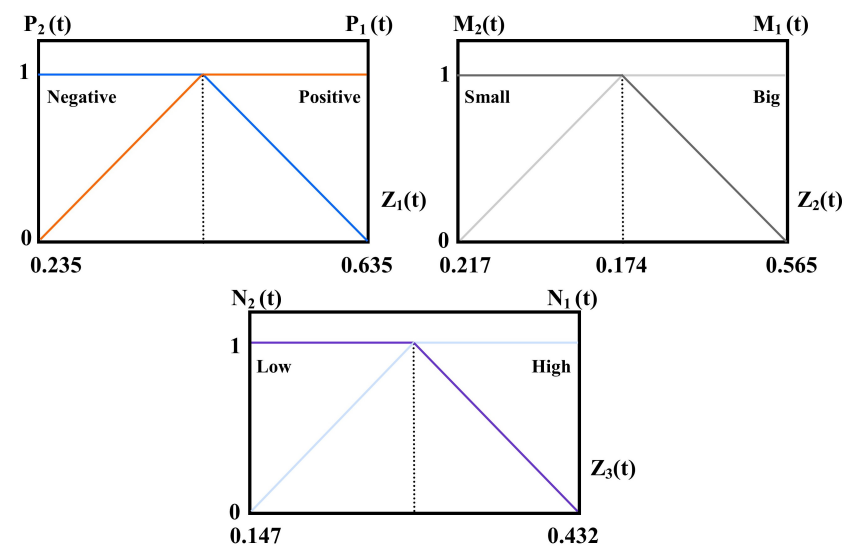

Figure 3: Membership functions of premise variables $z_{1}(t), z_{2}(t)$ and $z_{3}(t)$.

We define name of the membership functions "Positive", "Negative," "Big," and "Small," respectively. Figure 3 shows these membership functions. Here, we can generalize that the $i^{t h}$ rule of the continuous T-S fuzzy models are of the following forms:

\section{Model Rule i:}

$$
\begin{gathered}
\text { IF } z_{1}(t) \text { is } M_{i 1} \text { and } \cdots \text { and } z_{p}(t) \text { is } M_{i p}, \\
\text { THEN }\left\{\begin{array}{c}
\dot{x}=A_{i} x(t)+B_{i} u(t) \\
y(t)=C_{i} x(t)
\end{array}\right.
\end{gathered}
$$

Here, $M_{i j}$ is the fuzzy set and $\mathrm{r}$ is the number of model rules; $x(t)$ is the state vector, $u(t)$ is the input vector, $y(t)$ is the output vector, $A_{i}$ is the square matrix with real elements and $z_{1}(t), \cdots, z_{p}(t)$ are known premise variables as mentioned before. Each linear consequent equation represented by $A_{i} x(t)+B_{i} u(t)$ is called a subsystem.
Now, the nonlinear system (3) is modeled by the following fuzzy rules (we don't consider input $\mathrm{u}(\mathrm{t})$ ):

\section{Model Rule 1 to 8:}

IF $z_{1}(t)$ is "Positive" and $z_{2}(t)$ is "Big," and $z_{3}(t)$ is "High," THEN $x_{(}(t)=A_{1} x(t)$. IF $z_{1}(t)$ is "Positive" and $z_{2}(t)$ is "Big," and $z_{3}(t)$ is "Low," THEN $x_{(}(t)=A_{1} x(t)$. IF $z_{1}(t)$ is "Positive" and $z_{2}(t)$ is "Small," and $z_{3}(t)$ is "High," THEN $\left.x_{(} t\right)=A_{1} x(t)$. IF $z_{1}(t)$ is "Positive" and $z_{2}(t)$ is "Small," and $z_{3}(t)$ is "Low," THEN $x_{(}(t)=A_{1} x(t)$. IF $z_{1}(t)$ is "Negative" and $z_{2}(t)$ is "Big," and $z_{3}(t)$ is "High," THEN $x(t)=A_{1} x(t)$. IF $z_{1}(t)$ is "Negative" and $z_{2}(t)$ is "Big," and $z_{3}(t)$ is "Low," THEN $x_{(}(t)=A_{1} x(t)$.

IF $z_{1}(t)$ is "Negative" and $z_{2}(t)$ is "Small," and $z_{3}(t)$ is "High," THEN $x_{(}(t)=A_{1} x(t)$.

IF $z_{1}(t)$ is "Negative" and $z_{2}(t)$ is "Small," and $z_{3}(t)$ is "Low," THEN $x_{(}(t)=A_{1} x(t)$.

where the subsystems are determined as:

$$
A_{1}=\left[\begin{array}{ccc}
0.0066 & 0 & z_{1} \in \text { Positive } \\
& \max z_{3} & 0 \\
0.0114 & z_{3} \in \text { High } & \\
\max z_{2} & 0.05681 & 0.0132 \\
z_{2} \in \text { Big } &
\end{array}\right]
$$

$$
A_{8}=\left[\begin{array}{ccc}
0.0066 & 0 & z_{1} \in \text { Negative } \\
& \max z_{3} & 0 \\
0.0114 & z_{3} \in \text { Low } & \\
\max z_{2} & 0.05681 & 0.0132
\end{array}\right]
$$

which is

$$
\begin{aligned}
& A_{1}=\left[\begin{array}{ccc}
0.0066 & 0 & 0.635 \\
0.0114 & 0.432 & 0 \\
0.565 & 0.05681 & 0.0132
\end{array}\right] \\
& A_{8}=\left[\begin{array}{ccc}
0.0066 & 0 & 0.235 \\
0.0114 & 0.432 & 0 \\
0.217 & 0.05681 & 0.0132
\end{array}\right]
\end{aligned}
$$


This T-S fuzzy model can exactly represents the nonlinear system in the region $[0.235,0.635] \times$ $[0.217,0.565] \times[0.147,0.432]$, on the $z_{1}, z_{2}$, and $z_{3}$ space.

\subsection{FLC Design and Structure}

In this work we adopt T-S type FLC for the simplicity of controller structure and easiness for encounter the nonlinear behaviour of the system. To design the T-S type controller, the FLC considered here having three input taken, error $(e(k))$, rate of change of error $(\dot{e}(k))$ and residue $(r(k))$ respectively. The output of the FLC is $u_{2}(k)$ For each input we use seven fuzzy labels, Positive Very High (PVH), Very High (VH), High (H), Moderate (M), Negative (N), Negative High $(\mathrm{NH})$, and Negative Very High $(\mathrm{NVH})$, for fuzzification, The degree to which set they belong to is determined by membership function illustrated in Fig. 3. There are in total $7^{3}=343$ fuzzy rules because of each input having 7 linguistic variable.
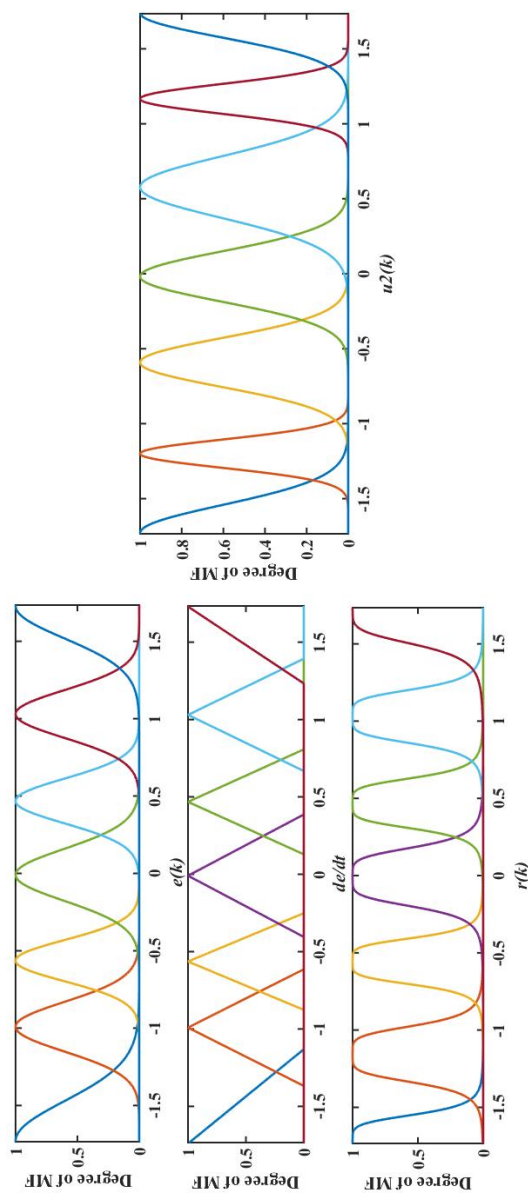

Figure 4: Membership function used for FLC.

A built-in membership function in the Matlab toolbox, named as Gaussian and triangle member- ship function are used to represent fuzzy set of $(e(k)),(\dot{e}(k)))$ and $r(k)$.

There are three reasons to employ the Gaussian and Triangle membership functions. First, the Gaussian and Triangle membership functions are appropriate to represent the concepts of positive and negative as well as gradually increasing and decreasing value. Second, the Gaussian membership function is exponential and Triangle membership functions are single-order polynomials, which are suitable for the implementation of the FLC because of their easiness in computing. Third, the FLC using the Gaussian and Triangle membership functions provides varying controller output based on the residua $(r(k))$ that are desirable for control of the height for three-tank benchmark system. The input output parameter range is found with operating point of three-tank system and normalized.

Each rule contributes to the final FLC output according to matching for the IF part of the fuzzy rule. The T-S type fuzzy inference takes a weighted average of the individual outputs for each rule. The output $\vartheta_{i}(i=1 \sim 343)$ for each rule is weighted by the firing strength $\mu_{R^{i}}$, which is calculated as given by equation 8. The structure of FLC is T-S type, which consists of rules of the following form

$$
\begin{gathered}
R^{i}: \operatorname{If}\left(e(k) \text { is } A_{1}^{i}\right) \operatorname{AND}\left(\dot{e}(k) \text { is } A_{2}^{i}\right) ; \\
A N D\left(r(k) \text { is } A_{3}^{i}\right), \operatorname{THEN}\left(u_{2}(k)=\vartheta_{i}\right)
\end{gathered}
$$

The firing strength for the first rule is

$$
\mu_{R^{2}}=\mu_{A_{1}}(e(k)) \mu_{A_{1}}(\dot{e}(k)) \mu_{A_{1}}(r(k))
$$

The final output of the fuzzy controller is calculated by aggregating all sixteen rules in the weighted form

$$
\mu_{f l c}=\frac{\sum_{i=1}^{343} \mu_{R^{i} u_{2}(k)}}{\sum_{i=1}^{343} \mu_{R^{i}}}
$$

\subsection{PID controller design}

The most common and simple control sachem is PID, which is used widely in industries because of its robustness and linear output[23].PID controller is an appropriate combination of proportional, integral and derivative terms to provide all the desired performances of a closed loop system.It also gives zero steady state error and overshoot is also very less. PID controller are recommended for use in slow processes and which are free from noise.The PID controller can be realized as a controller that takes account of the present, the past and the future of the 
error [34].Mathematically, PID controller is expressed as

$$
G_{c}(s)=K_{c}\left(1+\frac{1}{\tau_{i} s}+\tau_{d} s\right)
$$

Where, $K_{c}$ is proportional gain, $\tau_{i}$ id integral time and $\tau_{d}$ derivative time.

As PID controller has three parameters to be adjusted, so many different methods have been developed.Many researchers have proposed different tuning methods for tuning PID controller.In this article Ziegler \& Nichols tuning methods are used which is described below.

\section{Ziegler-Nichols Method}

In 1942, Ziegler and Nichols explained simple mathematical procedures for tuning PID controllers.These procedures are now accepted as standard in control systems practice [3]. They proposed a mathematical table for tuning each parameters of PID controller.They proposed different procedures for open loop and closed loop systems.

\section{Ziegler-Nichols Open-Loop Method}

In this method we have to obtain step response of open loop system, so it is also called process curve method.

Steps involved in tuning a process using this method [4] are

- Make an open-loop step test of process transfer function.

- From the process reaction curve, determine dead time $\left(\tau_{\text {dead }}\right)$, time constant $(\tau)$, ultimate value that the response reaches at steady state, $\left(M_{u}\right)$ for a step change of $\left(P_{0}\right)$.

$$
K_{0}=\frac{P_{0}}{M_{u}} * \frac{\tau}{\tau_{\text {dead }}}
$$

- Obtain PID tuning parameters using Table 2

\begin{tabular}{|c|c|c|c|}
\hline & $K_{c}$ & $\tau_{i}$ & tau $_{d}$ \\
\hline PID & $1.2 P_{0}$ & $2 \tau_{\text {dead }}$ & $0.5 \tau_{\text {dead }}$ \\
\hline
\end{tabular}

Table 2: Ziegler-Nichols Open-Loop Tuning Parameters [4].

Based on Ziegler-Nichols tuning parameters tabulated in table 2, the PID controller parameters are found as follows:

$$
K_{c}=1.5482, \tau_{i}=2.4616, \text { and } \tau_{d}=0.7812
$$

Now, proposed Passive FTC output $\left.u_{(} k\right)$ is combination of PID output $u_{1}(k)$ and Type- 1 FLC output $u_{2}(k)$ which is given by eq. 15 .

$$
u(k)=u_{1}(k)+u_{2}(k)
$$

\section{Simulation Results}

The results shown in the following figures are step responses obtained for specific operating points.

The application results are depicted for:

- System component fault (Tank bottom leak) (Abrupt in nature) at $\mathrm{t}=4 \mathrm{sec}$ with the PFTC control (figure 4)

- System component fault (Tank bottom leak) (Increasing in nature) at $\mathrm{t}=4 \mathrm{sec}$ with the PFTC control (figure 5)

- Pump fault (blocked or loss of effectiveness) (Abrupt in nature) at $\mathrm{t}=4 \mathrm{sec}$ with the PFTC control (figure 6)

- Pump fault (blocked or loss of effectiveness) (Increasing in nature) at $\mathrm{t}=4 \mathrm{sec}$ with the PFTC control (figure 7)

- System component (Tank bottom leak) and pump fault (blocked or loss of effectiveness) simultaneously occurs, the fault having in different in nature (abrupt and increasing) at $\mathrm{t}=4 \mathrm{sec}$ and $\mathrm{t}=12$ sec with the PFTC control (figure 8)

\subsection{PFTC in case of system component fault:}

On figure 5 , it can be seen that the system component fault (tank bottom leak) introduced at tank 3 at $\mathrm{t}=4$ sec. The different magnitude of leak fault introduced in to tank 3 with abrupt in nature. The result figures clearly shown the effects of leak fault on steady state. At the instant system faults occurs the controller law $u(k)$ given by T-S fuzzy logic control plus PID control which preserve the system performances at acceptable level.The control performance of the Passive FTC subject to system component fault (Abrupt nature) measured using IAE and ISE errors, the error results illustrated in table 3 .

On figure 6 , it can be seen that the system component fault (tank bottom leak) introduced at tank 3 at $\mathrm{t}=4$ sec.The different magnitude of leak fault introduced in to tank 3 with increasing in nature.The result figures clearly shown the effects of leak fault on steady state. At the instant system faults occurs the controller law $u(k)$ given by T-S fuzzy logic control plus PID control 


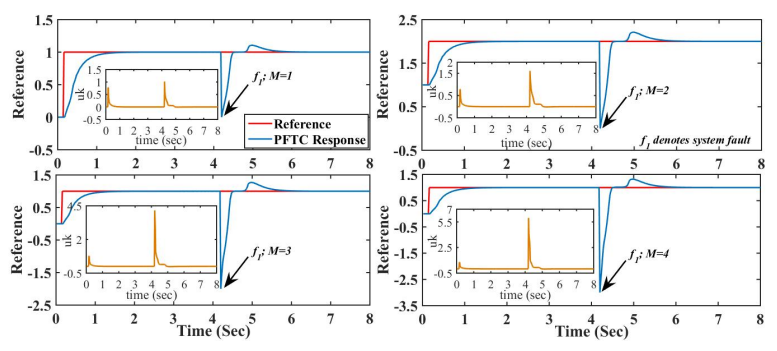

Figure 5: PFTC control response of system component fault abrupt in nature.

\begin{tabular}{|c|c|c|c|c|}
\hline Sr. No. & Controller & $f_{1}$ & IAE & ISE \\
\hline 1. & \multirow{3}{*}{ Passive } & $\mathrm{M}=1$ & 0.1606 & 0.0623 \\
\cline { 4 - 5 } \cline { 4 - 5 } FTC & $\mathrm{M}=2$ & 0.2142 & 0.1115 \\
\cline { 1 - 1 } & & $\mathrm{M}=3$ & 0.2445 & 0.1561 \\
\cline { 1 - 1 } 3. & $\mathrm{M}=4$ & 0.2698 & 0.2092 \\
\hline 4. & & \multicolumn{3}{|c}{} \\
\hline
\end{tabular}

Table 3: Integral error results of Passive FTC for system component fault abrupt in nature.

\begin{tabular}{|c|c|c|c|c|}
\hline Sr. No. & Controller & $f_{1}$ & IAE & ISE \\
\hline 1. & \multirow{4}{*}{ Passive } & $\mathrm{S}=1$ & 0.1213 & 0.04639 \\
\cline { 4 - 5 } & $\mathrm{S}=2$ & 0.1356 & 0.04782 \\
\cline { 4 - 5 } 2. & FTC & $\mathrm{S}=3$ & 0.152 & 0.0506 \\
\cline { 4 - 5 } 3. & & $\mathrm{~S}=4$ & 0.1675 & 0.05435 \\
\hline 4. & &
\end{tabular}

${ }^{*} \mathrm{~S}$ denotes the slope value for increasing fault nature.

Table 4: Integral error results of Passive FTC for system component fault increasing in nature.

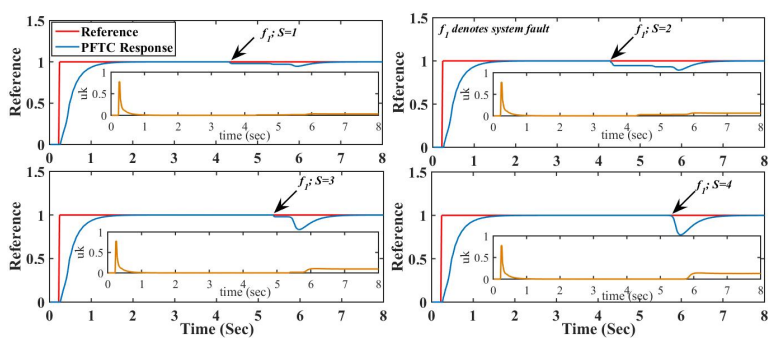

Figure 6: PFTC control response of system component fault increasing in nature.

which preserve the system performances at acceptable level.The control performance of the Passive FTC subject to system component faults with different magnitudes (increasing nature) illustrated in table 4 .

\subsection{PFTC in case of actuator fault}

On figure 7 , it can be seen that the actuator fault (block or loss of effectiveness) introduced for pump 1 at $\mathrm{t}=4 \mathrm{sec}$. The different magnitude of actuator faults introduced in to pump 1 with abrupt in
nature.The Passive FTC response clearly shows the effectiveness of the strategy. The controller law $u(k)$ smoothly maintain the reference point with all most zero steady state error.The control performance of the Passive FTC subject to actuator faults with different magnitudes (abrupt nature) illustrated in table 5 in terms of integral errors.
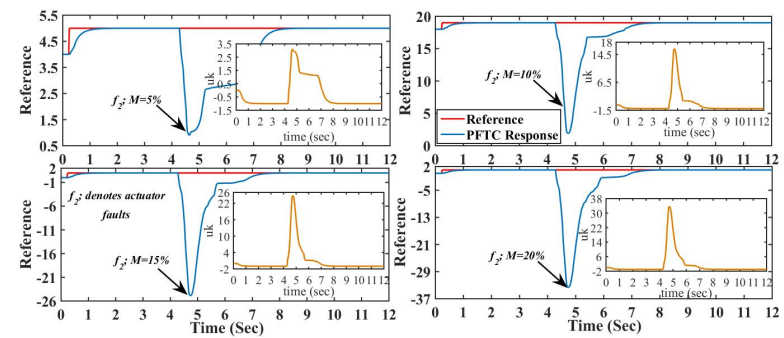

Figure 7: PFTC control response of actuator fault abrupt in nature.

\begin{tabular}{|c|c|c|c|c|}
\hline Sr.No. & Controller & $f_{2}$ & IAE & ISE \\
\hline 1. & \multirow{3}{*}{ Passive } & $\mathrm{M}=5 \%$ & 0.1213 & 0.04639 \\
\cline { 4 - 5 } & $\mathrm{M}=10 \%$ & 0.1356 & 0.04782 \\
\cline { 4 - 5 } 2. & $\mathrm{MTC}$ & $\mathrm{M}=15 \%$ & 0.152 & 0.0506 \\
\cline { 4 - 5 } 3. & & $\mathrm{M}=20 \%$ & 0.1675 & 0.05435 \\
\hline 4. & & \multicolumn{3}{|c}{} \\
\hline
\end{tabular}

Table 5: Integral error results of Passive FTC error result for actuator fault abrupt in nature.

\begin{tabular}{|c|c|c|c|c|}
\hline Sr. No. & Controller & $f_{2}$ & IAE & ISE \\
\hline 1. & \multirow{4}{*}{$\begin{array}{c}\text { Passive } \\
\text { FTC }\end{array}$} & $\mathrm{S}=1$ & 0.3291 & 0.1149 \\
\cline { 4 - 5 } \cline { 4 - 4 } & & $\mathrm{S}=2$ & 0.5514 & 0.3212 \\
\cline { 4 - 5 } & & $\mathrm{S}=3$ & 0.7737 & 0.6699 \\
\cline { 4 - 5 } 3. & $\mathrm{~S}=4$ & 0.996 & 1.144 \\
\hline 4. & &
\end{tabular}

Table 6: Integral error results of Passive FTC for actuator fault increasing in nature.

On figure 8, it can be seen that the actuator fault (block or loss of effectiveness) introduced for pump 1 at $\mathrm{t}=4 \mathrm{sec}$. The different magnitude of actuator faults introduced in to pump 1 with increasing in nature.The Passive FTC response clearly shows the effectiveness of the strategy. The control performance of the Passive FTC subject to actuator faults with different magnitudes (increasing nature) illustrated in table 6 in terms of integral errors.

\subsection{PFTC in case of actuator and system component faults simultaneously}

On figure 9, it can be seen that the system component (tank bottom leak) and actuator fault (block or loss of effectiveness) introduced simultaneously with different time. Also fault is introduce into system with 


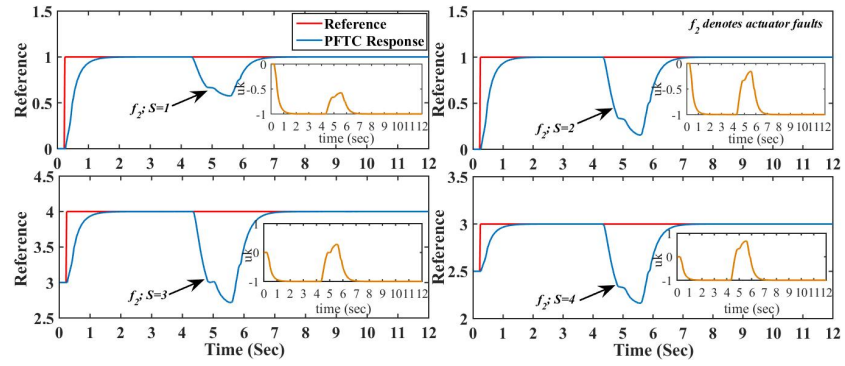

Figure 8: PFTC control response of actuator fault increasing in nature.
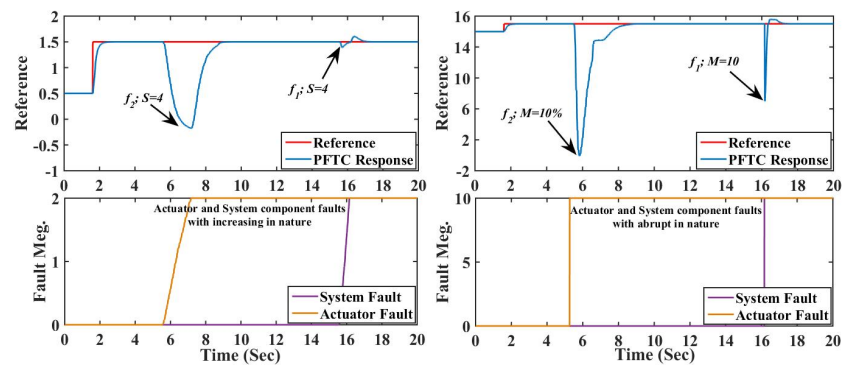

Figure 9: PFTC control response of system component and actuator fault simultaneously (abrupt and increasing in nature).

two different nature, one is abrupt and second is increasing. The system component (leak in tank 3 bottom) faults (increasing nature) introduced at $\mathrm{t}=16$ sec and actuator faults introduced at $t=6$ sec. At the other side to check response subject same faults but abrupt in nature introduced into the process system component fault at $\mathrm{t}=16 \mathrm{sec}$ and actuator faults at $\mathrm{t}=$ 6 sec.The critical observation and error results shows that, same magnitude actuator faults is degrade control performance drastically compared to same magnitude of system component faults for both the abrupt and increasing nature.Also from the responses we came to know, abrupt nature actuator and system component faults effects is more vulnerable compare to increasing nature. The comparative control performance of the Passive FTC subject to actuator faults with different magnitudes (increasing and abrupt nature) illustrated in table 7 in terms of integral errors.

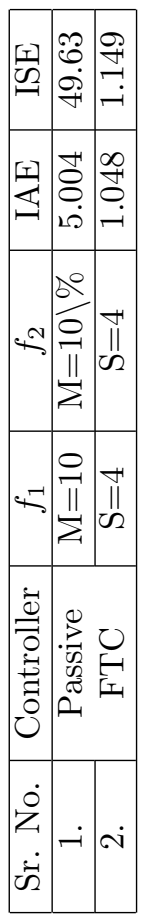

Table 7: Integral error results of Passive FTC result comparison for simultaneous system component and actuator fault (abrupt and increasing in nature).

\section{Conclusion}

The method developed in this paper allows to increase the application field of Fault Tolerant control law: the industrial processes without hardware redundancy, in the event of actuator or system component failures.A significant actuator failure (blocked or loss of effectiveness), system component fault (tank bottom or pipe leak) on a process without hardware redundancy can be considered as a critical failure.Its application to a non-linear process, a three-tank system, emphasizes the importance and usefulness of such a fault tolerance.The objective of this Fault Tolerant control is different from that usually met.Indeed, in the presence of critical faults on such process, it is impossible to maintain the damaged system at some acceptable level of performances, whatever the applied control strategy. The objective is so to operate safely and to minimize the loss of productivity. The abrupt and increasing nature of actuator and system component faults are taken to check the efficacy of the proposed Passive FTC scheme.It is realized from the simulation responses and error results T-S Fuzzy plus PID controller based Passive FTC is an efficient strategy for a non-linear three-tank level process.

In addition to this proposed work, Type- 2 FLC will be use full in the case of process having modeling uncer- 
tainty and unexpected noise is a major future scope of work.Also one o the key part of future work is to minimize computation time of Type-2 FLC.Future work is extended to optimization of Type-1 ant Type-2 fuzzy sets using Genetic Algorithm (GA) and design FLC using this membership functions, this approach was well defined in [16].

\section{Acknowledgement}

This research was carried out in Simulation lab ,Department of Instrumentation \& Control Engineering, Dharmsinh Desai University, Nadiad-Gujarat, India.We thank Dr.Jalesh Purohit, Professor in Chemical Engineering Department, Dharmsinh Desai University, Nadiad-Gujarat, India] for comments that greatly improved the manuscript. We would also like to thank "anonymous" reviewers for their so-called insights and constructive suggestions to enhance the quality of manuscript.We would also like to thank "anonymous" reviewers for their so-called insights and constructive suggestions to improve the quality of the paper.

\section{References}

[1] V. C. B. Jiang, M. Staroswiecki, Fault accommodation for a class of nonlinear dynamic systems, IEEE Transactions on automatic Control 51 (2006) 1578-1583.

[2] W. Bandler, L. J. Kohout, Fault tolerant control using interval type-2 takagi-sugeno fuzzy controller for nonlinear system, in: P. M. N. G. A. Abraham, A. Cherukuri (Ed.), Intelligent Systems Design and Applications, Vol. 941, Springer, Cham, Switzerland, 2020, pp. 150-164.

[3] J. C. Basilio, S. R. Matos, Design of pi and pid controllers with transient performance specification, IEEE Transactions on Education 45 (2002) $364-370$.

[4] B. W. Bequette, Process Control: Modelling, Design and Simulations, Prentice Hall, 2003.

[5] L. Cervantes, O. Castillo, Statistical comparison of type-1 and type-2 fuzzy systems design with genetic algorithms in the case of three tank water control, 2013 Joint IFSA World Congress and NAFIPS Annual Meeting (IFSA/NAFIPS) (2013) 1056-1061.

[6] O. C. . L. Cervantes, Genetic design of optimal type-1 and type-2 fuzzy systems for longitudinal control of an airplane, Intelligent Automation \& Soft Computing 20 (2013) 213-227.
[7] H. N. D. Theilliol, J. Ponsart, Fault diagnosis and accommodation of a three-tank-system based on analytical redundancy, ISA Transactions 41 (2002) 365-382.

[8] S. Ding, Model-based Fault Diagnosis Techniques-Design Schemes, Algorithms and tools, Springer-Verlag, London, 2008.

[9] J. Gertler, Fault Detection and Diagnosis in Engineering Systems, Taylor Francis, New York: Marcel Dekker, 1998.

[10] G.J.J.Ducard, Fault Tolerant Flight Control and Guidance Systems - Practical Methods for Small Unmanned Aerial Vehicles, Springer-Verlag, London, 2009.

[11] C. Hajiyev, F. Caliskan, Fault Diagnosis and Reconfiguration in Flight Control Systems, Kluwer Academic, Boston, 2003.

[12] R. Isermann, Fault-diagnosis Systems: An Introduction from Fault Detection to Fault tolerance, Springer, Berlin, Germany, 2006.

[13] J. Jiang, Y. Zhang, Accepting performance degradation in fault-tolerant control system design, IEEE Transactions on Control Systems Technology 14 (2006) 284-292.

[14] P. Kabore, H. Wang, Design of fault diagnosis filters and fault tolerant control for a class of nonlinear systems, IEEE Transactions on Automatic Control 11 (2002) 1805-1810.

[15] P. M. F. V. L. Cervantes, O. Castillo, Comparative study of type-1 and type-2 fuzzy systems for the three-tank water control problem, in: M. M. I. Batyrshin (Ed.), Advances in Computational Intelligence, Vol. 7630, Springer Heidelberg, Berlin, 2013, pp. 362-373.

[16] P. M. F. V. L. Cervantes, O. Castillo, Genetic optimization of membership functions in modular fuzzy controllers for complex problems, in: J. K. O. Castillo, P. Melin (Ed.), Recent Advances on Hybrid Intelligent Systems. Studies in Computational Intelligence, Vol. 451, Springer Heidelberg, Berlin, 2013, pp. 51-62.

[17] J. M. S. L. Mendonca, J. S. da Costa, Fault accommodation of an experimental three tank system using fuzzy predictive control, in: IEEE Proceedings of International Conference on Fuzzy Systems (IEEE World Congress on Computational Intelligence,Hong Kong, China), 2008, pp. 1619-1625. 
[18] P. Li, G. Yang, An adaptive fuzzy design for faulttolerant control of mimo nonlinear uncertain systems, Control Theory Applications 9 (2011) 244250 .

[19] J. L. M. Blanke, M. Kinnaert, M.Staroswiecki, Diagnosis and Fault-tolerant Control, 2nd Edition, Springer-Verlag, London, 2006.

[20] J. J. M. Mahmoud, Y. Zhang, Active fault tolerant control systems: Stochastic analysis and synthesis, in: Lecture Notes in Control and information Sciences. Proc. 5th EUSFLAT Conference, Vol. 287, Springer-Verlag Berlin Heidelberg, 2003, pp. 1-210.

[21] H. R. Patel, V. A. Shah, Fault detection and diagnosis methods in power generation plants - the indian power generation sector perspective: An introductory review, Journal of Energy and Management 2(2) (2018) 31-49.

[22] H. R. Patel, V. A. Shah, A fault-tolerant control strategy for non-linear system: An application to the two tank canonical noninteracting level control system, in: in Proc. IEEE Distributed Computing, VLSI, Electrical Circuits and Robotics (DISCOVER-2018), IEEE, Mangalore, India, 2018, pp. 64-70.

[23] H. R. Patel, V. A. Shah, Fault tolerant control systems: A passive approaches for single tank level control system, i-manager's Journal on Instrumentation and Control Engineering 6(1) (2018) 11-18.

[24] H. R. Patel, V. A. Shah, A framework for fault-tolerant control for an interacting and noninteracting level control system using ai, In Proceedings of the 15th International Conference on Informatics in Control, Automation and Robotics I (2018) 180-190.

[25] H. R. Patel, V. A. Shah, Fuzzy logic based passive fault tolerant control strategy for a singletank system with system fault and process disturbances, in: in Proc. 5th International Conference on Electrical and Electronics Engineering (ICEEE-2018), IEEE, Istanbul, Turkey, 2018, pp. $257-262$.

[26] H. R. Patel, V. A. Shah, Actuator and system component fault tolerant control using interval type-2 takagi-sugeno fuzzy controller for hybrid nonlinear process, International Journal of $\mathrm{Hy}$ brid Intelligent Systems. Pre-press (2019) 1-11.
[27] H. R. Patel, V. A. Shah, Fault tolerant controller using interval type-2 tsk logic control systems: Application to three interconnected conical tank system, 38th North American Fuzzy Information Processing Society Annual Conference. (to be published).

[28] H. R. Patel, V. A. Shah, Passive fault tolerant control system for two-tank interacting conical level control system against partial actuator failures, Journal of Computer Science. (to be published).

[29] H. R. Patel, V. A. Shah, Passive fault tolerant controller using hybrid controller for effective control of two tank canonical non-interacting system, Journal of Mathematics and Statistics. (to be published).

[30] H. R. Patel, V. A. Shah, Performance comparison of passive fault tolerant control strategy with pi and fuzzy control of single-tank level process with sensor and system fault, American Journal of Engineering and Applied Sciences. (to be published).

[31] H. R. Patel, V. A. Shah, Shah.," a passive faulttolerant control strategy for non-linear system: An application to the two tank conical noninteracting level control system, MASKAY 9(1) (2019) 1-8.

[32] R. Patton, Fault-tolerant control: The 1997 situation, IFAC Proceedings Volumes 30 (1997) 10331055.

[33] B. K. S. Nagarajan, Control of interacting level process under sensor failure conditions using coactive adaptive neuro-fuzzy observer, International Journal of Technologies in Science and Engineering 2 (2015) 242-251.

[34] C. A. Smith, A. B. Corripio, Principles and Practice of Automatic Process Control, John Wiley Sons, Inc., 2005.

[35] J. J. W. Chen, Fault-tolerant control against stuck actuator faults, in: IEEE Proceedings of Control Theory Applications, Vol. 152, 2005, pp. 138-146.

[36] J. J. Y.M. Zhang, D. Theilliol, Incorporating performance degradation in fault tolerant control system design with multiple actuator failures, International Journal of Control, Automation, and Systems 6 (2008) 327-338.

[37] L. A. Zadeh, The concept of a linguistic variable and its application to approximate reasoning I, Information Sciences 8 (1975) 199-250. 
[38] Y. Zhang, J. Jiang, Bibliographical review on reconfigurable fault-tolerant control systems, Annual Reviews in Control 32 (2008) 229-252. 